\title{
Ultrasensitive surface-enhanced Raman scattering nanosensor for mercury ion detection based on functionalized silver nanoparticles $\uparrow$
}

\author{
Lingxin Chen, ${ }^{\star a}$ Nan Qi, ${ }^{\text {ab }}$ Xiaokun Wang, ${ }^{\text {ac }}$ Ling Chen, ${ }^{\text {ad }}$ Huiyan You*b and Jinhua $\mathrm{Li}^{\text {a }}$
}

In this work, a simple, rapid and ultrasensitive surface-enhanced Raman scattering (SERS) nanosensor was developed for mercury ion $\left(\mathrm{Hg}^{2+}\right)$ detection based on 4-mercaptopyridine (4-MPY) functionalized silver nanoparticles (AgNPs) (4-MPY-AgNPs) in the presence of spermine. Here, the spermine would bind AgNPs through Ag-N bonds and induce remarkable aggregation of AgNPs, and thereby would generate significantly enhanced Raman intensity of the reporter molecule 4-MPY. Followed by the addition of $\mathrm{Hg}^{2+}$, the formation of the $\mathrm{Hg}-\mathrm{Ag}$ alloy blocked the adsorption of 4-MPY and spermine, resulting in the dispersion of 4-MPY-AgNPs and thus decreasing the Raman intensity, by which the $\mathrm{Hg}^{2+}$ could be sensed by SERS. A good linearity was obtained in the range of $1-100 \mathrm{nM}\left(R^{2}=0.987\right)$, and the relative standard deviation was between 0.85 and $5.50 \%$. The spermine-induced accumulation of 4-MPY-AgNPs largely enhanced the SERS responses, leading to a high detectability up to $0.34 \mathrm{nM}$. A real water sample with spiked $\mathrm{Hg}^{2+}$ was also analyzed, presenting satisfactory recoveries ranging from 94.5 to $108.5 \%$, confirming the practicability of the SERS nanosensor based method.

Received 11th December 2013 Accepted 13th March 2014

DOI: $10.1039 / c 3 r a 47492 e$

www.rsc.org/advances spectrometries. ${ }^{8}$ However, most of them are confronted with some limitations with respect to complex instruments, procedures or costs. Currently, nanomaterials, of various sizes, shapes, and compositions, especially, gold nanoparticles (AuNPs) and silver nanoparticles (AgNPs) have been widely used for construction of nanosensors, since they often exhibit high extinction coefficient in the visible region and unique colortunable behavior that mainly depend on the interparticle distance. ${ }^{11-14}$ In recent years, several groups have proposed various detection methods for $\mathrm{Hg}^{2+}$ based on AuNPs and AgNPs. Liu's group has developed a one-step, room temperature, colorimetric method by using oligonucleotide-tethered AuNPs probes and a linker oligonucleotide with a number of T-T mismatches, resulting in the formation of particle aggregates accompanied colorimetric response with the addition of $\mathrm{Hg}^{2+}$ into the solution. ${ }^{15}$ Rex et al. have provided a sensitive assay through pushing the limits of $\mathrm{Hg}^{2+}$ sensors with gold nanorods, taking advantage of the strong affinity between $\mathrm{Au}$ and $\mathrm{Hg} .{ }^{16}$ Our group has also reported blue-to-red colorimetric sensing strategy for $\mathrm{Hg}^{2+}$ and $\mathrm{Ag}^{+}$via redox-regulated surface chemistry of AuNPs. ${ }^{17}$ Although the developed colorimetric methods possess good sensitivity and selectivity, it is still highly desirable to explore simpler and more sensitive methods for the detection of $\mathrm{Hg}^{2+}$.

Surface-enhanced Raman scattering (SERS), an ultrasensitive vibrational spectroscopic technique, has attracted lots of attentions due to its evading photobleaching, narrow bandwidth as well as the ability to detection of multiple analytes by a single laser wavelength. Many researches have reported on the SERS theory and it's commonly considered that two primary 
mechanisms contribute to the enhancement, long-range electromagnetic enhancement and short-range chemical enhancement. ${ }^{18-20}$ Since its discovery in late 1970 s, various types of SERS nanoprobes have been developed..$^{21-25}$ For example, Zhan's group has provided an AgNPs aggregates-based SERS substrate for sensitive detection of polycyclic aromatic hydrocarbons. ${ }^{23}$ Kuang's group has constructed a simple but highly sensitive SERS sensor platform based on a self-assembled gold nanostar dimer for $\mathrm{Hg}^{2+}$ detection. ${ }^{24}$ Our group has also developed two SERS strategies for highly sensitive detection of $\mathrm{As}^{3+}$ ions ${ }^{21}$ and $\operatorname{trypsin}^{25}$ using glutathione functionalized AgNPs and 4-mercaptopyridine (4-MPY)-functionalized AgNPs, respectively.

Inspired by these studies, herein, we proposed to develop a sensitive and selective SERS approach for $\mathrm{Hg}^{2+}$ detection based on 4-MPY functionalized AgNPs with the aid of redox-regulated surface chemistry of AgNPs. Spermine could induce the aggregation of 4-MPY functionalized AgNPs through Ag-N bonds, ${ }^{26}$ and thus, in the presence of spermine, significant SERS enhancement of Raman report molecule 4-MPY was obtained. However, with the addition of $\mathrm{Hg}^{2+}$, the spermine-induced aggregation was disturbed, which may well be mainly due to that the interaction between $\mathrm{Hg}^{2+}$ and AgNPs could occur in a short time. ${ }^{27}$ The resultant $\mathrm{Hg}-\mathrm{Ag}$ alloy on the surface of AgNPs could affect the adsorption of spermine and 4-MPY, and thereby would result in the decreased of Raman signals. Several major influence parameters of the SERS intensity were investigated, and under optimized conditions, excellent analytical performances were attained such as high sensitivity, high precision and high selectivity. The SERS nanosensor method was further demonstrated potentially applicable for monitoring $\mathrm{Hg}^{2+}$ in real water samples.

\section{Experimental}

\subsection{Reagents}

Silver nitrate $\left(\mathrm{AgNO}_{3}, 99.8 \%\right)$ and sodium hydroxide $(\mathrm{NaOH}$, 96\%) were obtained from Sinopharm Chemical Reagent Co., Ltd. (Shanghai, China), hydroxylamine hydrochloride $\left(\mathrm{NH}_{2} \mathrm{OH} \cdot \mathrm{HCl}\right)$ was purchased from Tianjin Kermel Chemical Reagent Co., Ltd. (Tianjin, China) and used as received. 4Mercaptopyridine (4-MPY, 95\%) and spermine (97\%) were obtained from Sigma-Aldrich (USA). Mercury nitrate $\left(\mathrm{Hg}\left(\mathrm{NO}_{3}\right)_{2}\right)$ was purchased from Sinopharm Group Chemical Reagent Co., Ltd. (Beijing, China). All other solvents and reagents used were of analytical grade.

\subsection{Instrumentation}

SERS spectra were recorded using a Thermo Scientific RFS100 Raman system equipped with a microscope and a $632.8 \mathrm{~nm}$ diode pumped He-Ne laser source. The transmission electron microscopy (TEM) analyses were obtained using a JEM1230 electron microscope (JEOL, Ltd., Japan) operating at 100 $\mathrm{kV}$. Dynamic light scattering (DLS) measurements were performed on a Malvern Zetasizer Nano-ZS90 (ZEN3590, UK). ICP-MS analyses were performed on PerkinElmer Elan DRC II (USA). Solutions throughout the work were prepared using fresh double deionized water, which was produced by a Cascada TM LS Ultrapure water system (Pall Corp., USA) with the water outlet operating at $18.2 \mathrm{M} \Omega$. All glassware in this work were soaked and washed with freshly prepared aqua regia, rinsed thoroughly in double deionized water and dried in air for use.

\subsection{Functionalization of AgNPs with 4-MPY}

AgNPs were synthesized referring to our previous work ${ }^{21}$ with necessary modification by reducing silver nitrate using hydroxylamine hydrochloride at room temperature. First, 1 $\mathrm{mL}$ of $0.30 \mathrm{M} \mathrm{NaOH}$ solution was added to $89 \mathrm{~mL}$ of $1.50 \mathrm{mM}$ $\mathrm{NH}_{2} \mathrm{OH} \cdot \mathrm{HCl}(10.44 \mathrm{mg})$ solution to maintain an alkaline $\mathrm{pH}$. Next, $10 \mathrm{~mL}$ of $0.01 \mathrm{M} \mathrm{AgNO}_{3}$ solution was added to the above solution with continuous stirring. The reaction mixtures were continuously stirred for an additional $0.5 \mathrm{~h}$. The prepared silver colloid was stored at room temperature. Then, $20 \mu \mathrm{L}$ of $400 \mu \mathrm{M} 4$-MPY was added to $2.7 \mathrm{~mL}$ of the above prepared silver colloid with stirring for $0.5 \mathrm{~h}$. Finally, the resultant mixtures were 4-MPY functionalized AgNPs (4-MPY-AgNPs).

\subsection{SERS detection of $\mathbf{H g}^{2+}$}

$20 \mu \mathrm{L}$ of spermine was added to $100 \mu \mathrm{L}$ of Tris-HCl buffer solution ( $\mathrm{pH}$ 8.0, $0.05 \mathrm{mM}$ ) containing 4-MPY functionalized AgNPs, and the resultant solutions were incubated for $2 \mathrm{~min}$, followed by adding $\mathrm{Hg}^{2+}$ solutions from 10 to $90 \mu \mathrm{L}$. The obtained mixtures were incubated at room temperature for 5 min. Then the samples were detected by Raman spectrometry with an exposure time of $4 \mathrm{~s}$.

\subsection{Selectivity study}

A series of metal ion solutions including $\mathrm{Pb}^{2+}, \mathrm{Cd}^{2+}, \mathrm{K}^{+}, \mathrm{Ca}^{2+}$, $\mathrm{Mg}^{2+}, \mathrm{Cu}^{2+}, \mathrm{Co}^{2+}, \mathrm{Na}^{+}, \mathrm{Mn}^{2+}, \mathrm{Cr}^{3+}, \mathrm{Fe}^{3+}, \mathrm{Zn}^{2+}$ and $\mathrm{MeHg}^{+}$were prepared and then added to the system to investigate the selectivity of this system toward $\mathrm{Hg}^{2+}$ under the same optimized conditions.

\subsection{Analysis of real water samples}

Tap water samples were collected from our institute and analyzed without treatment. A series of samples were spiked with standard $\mathrm{Hg}^{2+}$ solutions at certain concentrations. These spiked samples were added to a 4-MPY functionalized AgNPs containing spermine and $0.05 \mathrm{mM}$ Tris-HCl buffer solution $(\mathrm{pH}$ 8.0). The obtained mixtures were incubated at room temperature for $5 \mathrm{~min}$ and then measured by SERS.

\subsection{Safety consideration}

Aqua regia has strong oxidizing capacity and adverse effects on human health, thus all of the experiments involving aqua regia should be performed with gloves and protective glasses. The waste solution of the experiment should be collectively reclaimed to avoid polluting the environment. 


\section{Results and discussion}

\subsection{Analytical principle}

Scheme 1 shows the possible analytical principle of the detection of $\mathrm{Hg}^{2+}$ based on SERS by using AgNPs as enhancement substrates. Hydroxylamine chloride ions stabilized AgNPs were well dispersed (Fig. 1A(a)) and presented a bright yellow color (Fig. 1B(a)). In the process of SERS probe synthesis, with the addition of proper amount of Raman reporters 4-MPY, AgNPs were still a bright yellow color (Fig. 1B(b)), which indicated that the 4-MPY functionalized AgNPs remained monodisperse state (Fig. $1 \mathrm{~A}(\mathrm{~b})$ ). It was because that 4-MPY replaced parts of the hydroxylamine ions, and then both 4-MPY and hydroxylamine ions were present on the surface of AgNPs. Consequently, 4-MPY functionalized AgNPs could have strong Raman signals, and the presence of hydroxylamine ions could keep the stability of AgNPs. As a rule, 4-MPY combines with AgNPs through three different ways, Ag-S bonds, $\mathrm{Ag}-\mathrm{N}$ bonds, or via the aromatic $\pi$ electrons. ${ }^{25}$ It could deduce that the 4-MPY molecules adsorbed on the AgNPs surface were mainly through Ag-S bonds, while Ag$\mathrm{N}$ bonds played a minor role and the surface-aromatic $\pi$ interaction was not very important. ${ }^{25,28,29}$ When spermine was added to the 4-MPY-AgNPs solution, remarkable aggregation of the 4MPY-AgNPs occurred due to the distinctive structure of spermine. Since there are coordinating interactions between $\mathrm{N}$ and the electron-deficient surface of the metal nanoparticles, it makes compounds with electron-rich $\mathrm{N}$ easily bind onto the surface of metal nanoparticles. Hence, the $\mathrm{N}$-donors have a stronger affinity with AgNPs. ${ }^{26,30,31}$ So, in the presence of spermine, the formation of $\mathrm{Ag}-\mathrm{N}$ bonds led to remarkable aggregation of the 4-MPY-AgNPs (Fig. 1A(c)) along with an obvious color change from bright yellow to brown (Fig. 1B(c)). Meanwhile, the aggregated 4-MPY-AgNPs could produce a number of "hot spots", and therefore the Raman scattering signals of 4-MPY were greatly enhanced (Fig. 2, curve h). However, interestingly, once addition of $\mathrm{Hg}^{2+}$ to the system, the SERS signals would be remarkably reduced (Scheme 1) and hence the $\mathrm{Hg}^{2+}$ ions could be sensed optically. The redox-regulated surface chemistry between $\mathrm{Hg}^{2+}$

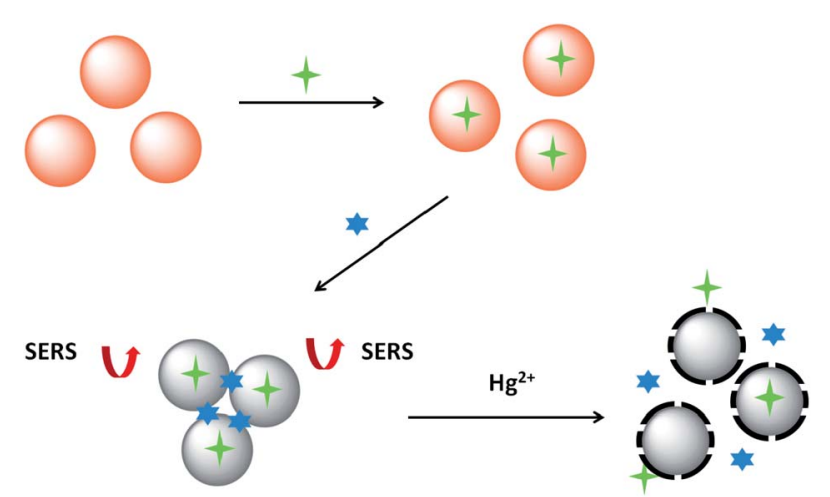

Ag NPs

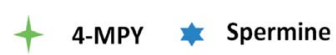

$\bigcirc \mathrm{Hg}-\mathrm{Ag}$ alloy

Scheme 1 Schematic of the SERS sensing principle for $\mathrm{Hg}^{2+}$ detection based on 4-MPY functionalized AgNPs.
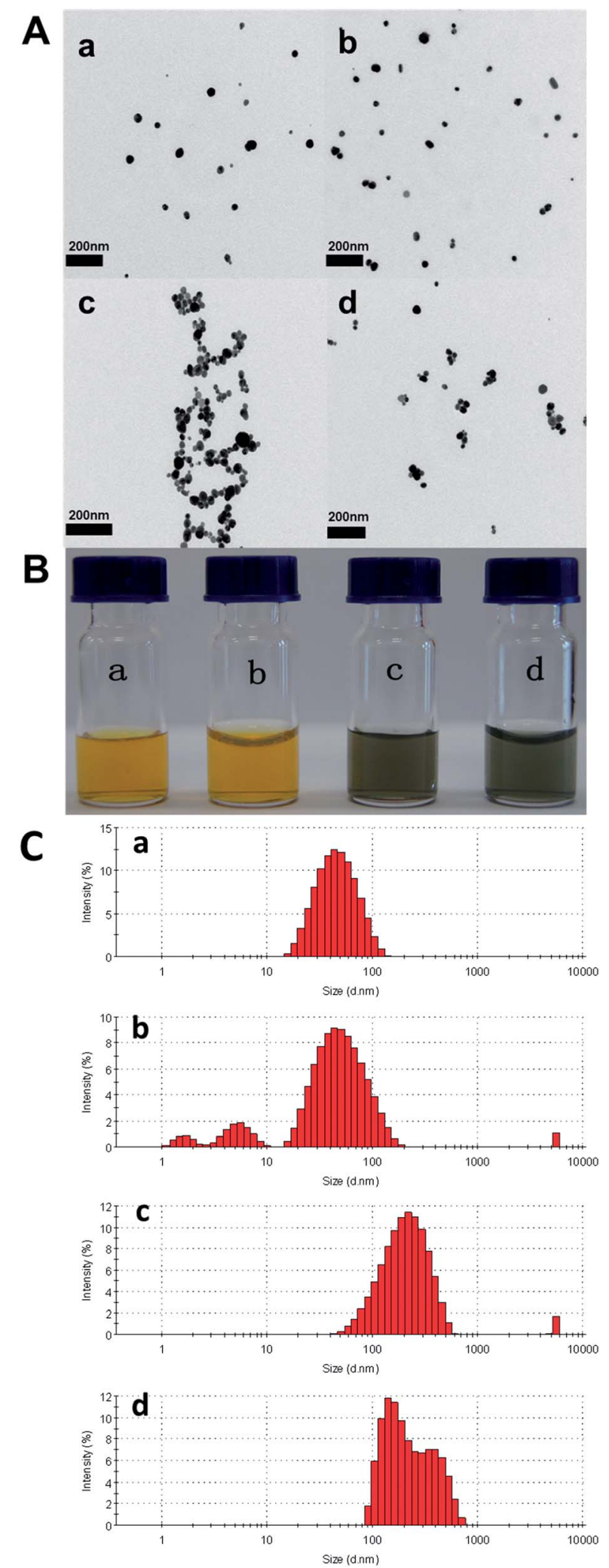

Fig. 1 TEM images (A), color changes (B) and size distributions (C) corresponding to (a) AgNPs, (b) 4-MPY-AgNPs, (c) 4-MPY-AgNPs + spermine, and (d) 4-MPY-AgNPs + spermine $+\mathrm{Hg}^{2+}$. 


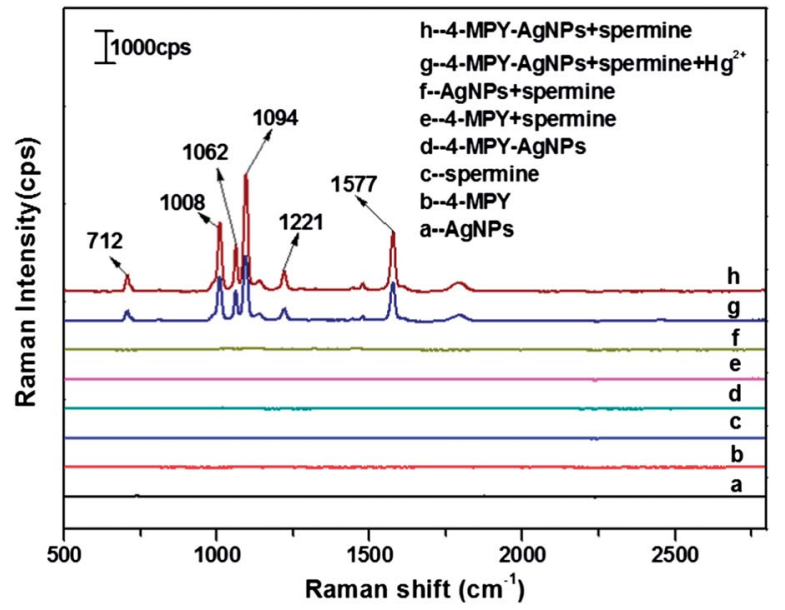

Fig. 2 Raman spectra of (a) AgNPs, (b) 4-MPY, (c) spermine, (d) 4MPY-AgNPs, (e) 4-MPY + spermine, (f) AgNPs + spermine, (g) 4-MPYAgNPs + spermine $+\mathrm{Hg}^{2+}$ and (h) 4-MPY-AgNPs + spermine.

and AgNPs would cause the formation of $\mathrm{Hg}-\mathrm{Ag}$ alloys and thereby non-uniform $\mathrm{Hg}-\mathrm{Ag}$ shells would wrap around the AgNPs. ${ }^{27,32}$ ICP-MS was used to provide a further evidence for the $\mathrm{Hg}-\mathrm{Au}$ alloys, as seen in Fig. S1 in the ESI. $\dagger$ Therefore, the adsorption of 4-MPY and spermine on the AgNPs surface was hindered and then it was unfavorable for the aggregation of 4-MPY-AgNPs (Fig. 1A(d)), as illustrated in Scheme 1.

Moreover, the size distributions of particles were measured by dynamic light scattering (DLS). It tends to overestimate the diameters of AgNPs measured by TEM images because DLS measures hydrodynamic diameters of silver nanocomposites. ${ }^{33} \mathrm{~A}$ pronounced difference in the AgNPs at different conditions displayed the different dominant distribution peaks, as shown in Fig. 1C. It was observed that the dominant distribution peak around $50 \mathrm{~nm}$ was for AgNPs (a), $57 \mathrm{~nm}$ for 4-MPY-AgNPs (b), $222 \mathrm{~nm}$ for spermine-induced aggregated 4-MPY-AgNPs (c), and $178 \mathrm{~nm}$ for the aggregated 4-MPY-AgNPs with the presence of $\mathrm{Hg}^{2+}$ (d), respectively. Even if the DLS signals were spread over a relatively large size distribution, the mean diameter agreed with the mean diameter obtained by TEM. As a result, the aggregation degree could be quantitatively estimated by determining the size distribution by DLS. Consequently, the degree of SERS intensity decrease could be employed for analytical detection of $\mathrm{Hg}^{2+}$.

In order to confirm the feasibility of the SERS sensing for $\mathrm{Hg}^{2+}$ detection, a series of control experiments were implemented and the results were shown in Fig. 2. It could be seen that in the presence of separate AgNPs, 4-MPY, spermine, or any two of them, there were no obvious SERS signals (Fig. 2, curve af). Excitedly, several strong signal peaks were obtained in the simultaneous presence of AgNPs, 4-MPY $(3.0 \mu \mathrm{M})$ and spermine $(140 \mu \mathrm{M})$, as exhibited in Fig. 2 (curve h). However, interestingly, upon addition of $\mathrm{Hg}^{2+}(10 \mathrm{nM})$, all SERS peaks significantly decreased (Fig. 2, curve g), which indicated that the formation of $\mathrm{Hg}-\mathrm{Ag}$ alloy and thereby $\mathrm{Hg}-\mathrm{Ag}$ shells wrapping around AgNPs destroyed the aggregation of 4-MPY-AgNPs. All the results demonstrated the proposed SERS strategy was feasible to $\mathrm{Hg}^{2+}$ analysis.

\subsection{Parameter optimization}

In order to acquire satisfactory SERS intensity and thereby analytical results, several parameters were optimized. Firstly, the effect of $\mathrm{pH}$ values (pH 7.0-9.0) in $0.05 \mathrm{mM}$ Tris-HCl buffer solution on SERS signal intensity was studied. As seen from Fig. S2A, $\uparrow$ the SERS signal was maximized at $\mathrm{pH} 8.0$ and the particles could keep stable, while at $\mathrm{pH}>8.0$, the SERS signal presented a significant decrease. Thus, a pH 8.0 Tris-HCl buffer solution was selected for the system. Next, the effect of the concentration of 4-MPY on SERS signal intensity was investigated. Six different concentrations of 4-MPY ranging from 1.0 to $3.5 \mu \mathrm{M}$ were tested, and the corresponding Raman spectra were recorded in Fig. S2B. $\uparrow$ It was observed that the SERS signal intensity increased with the concentration increase of 4-MPY, and the largest signal was achieved with $3.0 \mu \mathrm{M}$ 4-MPY. However, further increasing the concentration of 4-MPY resulted in SERS signal decrease. Thus, the concentration of $3.0 \mu \mathrm{M}$ was chosen in this work. The influence of the concentration of spermine on the SERS intensity was further investigated. As shown in Fig. S2C, $\dagger$ the spermine at the concentration lower than $140 \mu \mathrm{M}$ would be less efficient and unfavorable to SERS signals, whereas higher concentration than $140 \mu \mathrm{M}$ could not induce obvious enhancement of SERS signal. In order to ensure the full combination of AgNPs and spermine, the concentration of $140 \mu \mathrm{M}$ was adopted.

\subsection{Analytical sensitivity}

In order to evaluate the detectability of the developed SERS method, under the above optimized conditions, the SERS spectra of 4-MPY on AgNPs to different concentrations of $\mathrm{Hg}^{2+}$ were recorded for sensitivity investigation. As shown in Fig. 3A, there are many spectral features that are characteristics of 4-MPY, such as those at $712,1008,1062,1094,1221$ and $1577 \mathrm{~cm}^{-1}$. It could be clearly seen that the Raman intensity of all the characteristic peaks decreased with the increase of the concentration of $\mathrm{Hg}^{2+}$. The peak at $1094 \mathrm{~cm}^{-1}$ corresponding to the ring-breath/C-S stretching mode indicated that 4-MPY was bound to the surface of AgNPs through the $S .^{34}$ Also, the $1094 \mathrm{~cm}^{-1}$ peak was found to be the most prominent one, and its intensity was very sensitive to the concentration of $\mathrm{Hg}^{2+}$. Therefore, the Raman intensity of 4-MPY on AgNPs at $1094 \mathrm{~cm}^{-1}$ was used here for quantitative analysis of $\mathrm{Hg}^{2+}$. As displayed in Fig. 3B, a good linearity was obtained between the Raman intensity and the logarithm of the $\mathrm{Hg}^{2+}$ concentration within 1-100 $\mathrm{nM}\left(R^{2}=0.987\right)$, with relative standard deviations ranging from 0.85 to $5.50 \%$. The limit of detection based on a signal-to-noise ratio of 3 was estimated to be $0.34 \mathrm{nM}$, which was lower than the maximum contaminant level for $\mathrm{Hg}^{2+}$ in drinking water, namely $10 \mathrm{nM}$ defined by EPA. Therefore, the SERS strategy was indicated highly sensitive and reliable for the detection of $\mathrm{Hg}^{2+}$.

\subsection{Analytical selectivity}

In order to demonstrate the analytical selectivity of the SERS system toward $\mathrm{Hg}^{2+}$, other metal ions were introduced for investigation, including $\mathrm{Pb}^{2+}, \mathrm{Cd}^{2+}, \mathrm{K}^{+}, \mathrm{Ca}^{2+}, \mathrm{Mg}^{2+}, \mathrm{Cu}^{2+}, \mathrm{Co}^{2+}$, 
A
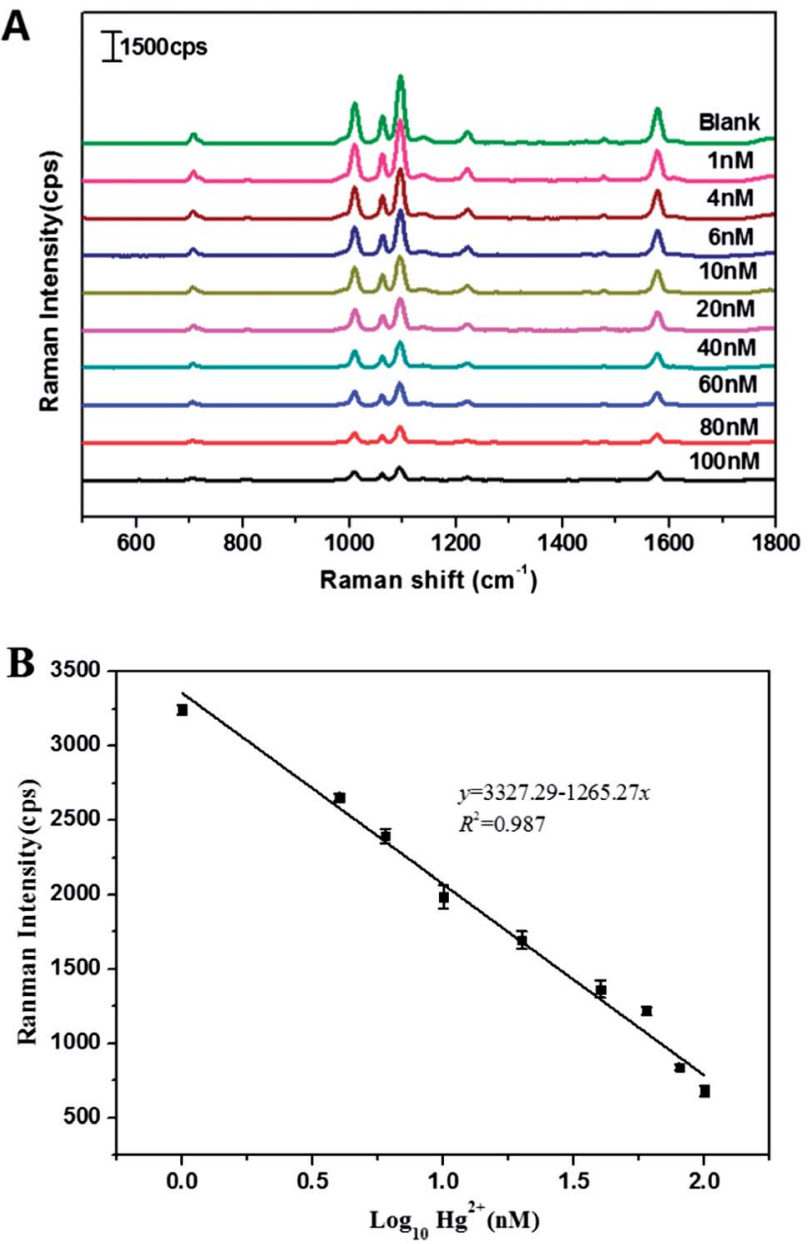

Fig. 3 (A) SERS spectra changes of 4-MPY functionalized AgNPs with different concentrations of $\mathrm{Hg}^{2+}(0-100 \mathrm{nM})$. (B) A plot of corresponding intensity of the Raman band at $1094 \mathrm{~cm}^{-1}$ versus $\mathrm{Hg}^{2+}$ concentrations in the range of 1 to $100 \mathrm{nM}\left(R^{2}=0.987\right)$. The error bars represent the standard deviations based on three independent measurements.

$\mathrm{Na}^{+}, \mathrm{Mn}^{2+}, \mathrm{Cr}^{3+}, \mathrm{Fe}^{3+}, \mathrm{Zn}^{2+}$ and $\mathrm{MeHg}^{+}$. As shown in Fig. 4, the SERS intensity ratio presented the maximum value for $\mathrm{Hg}^{2+}$. It was also observed that several other metal ions presented some signals, which was very likely owing to the interaction between the metal ions and 4-MPY as well as the ionic strength effects. Nevertheless, this signal change induced by $100 \mathrm{nM}$ of other metal ions was much smaller comparing with the response to $5 \mathrm{nM} \mathrm{Hg}^{2+}$. Besides, the SERS ratio value for the $\mathrm{Hg}^{2+}$ in the presence of a mixture of all the tested other metal ions was found closer to that for the $\mathrm{Hg}^{2+}$ individual, as evidenced in Fig. 4. These results indicated that the other metal ions had no significant effect on the $\mathrm{Hg}^{2+}$ detection. So, the developed SERS strategy was highly selective toward $\mathrm{Hg}^{2+}$.

\subsection{Practical application}

To evaluate the application of the presented assay in real samples, we utilized it to analyze $\mathrm{Hg}^{2+}$ in tap water samples by using standard addition method. As shown in Table 1, satisfying recoveries were obtained in a range of $94.5-108.5 \%$ for the

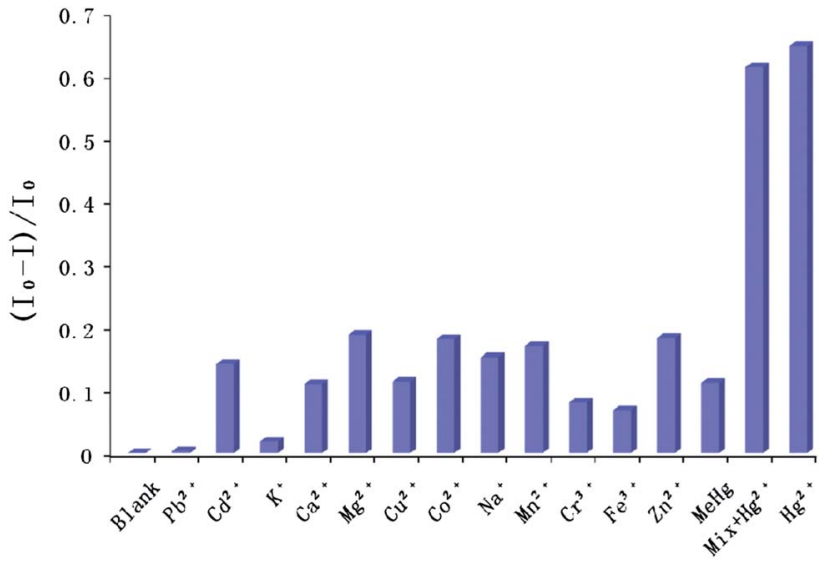

Fig. 4 Relative SERS intensity change of 4-MPY functionalized AgNPs at $1094 \mathrm{~cm}^{-1}$ upon the addition of various metal ions. $I_{0}$ is the SERS intensity of 4-MPY-AgNPs in the presence of spermine, and $/$ is the SERS intensity in the presence of other metal ions or $\mathrm{Hg}^{2+}$. The concentrations of all the metal ions were individual at $100 \mathrm{nM}$, except $\mathrm{Hg}^{2+}$ at $5 \mathrm{nM}, \mathrm{MeHg}^{+}$at $10 \mathrm{ppb}(46.38 \mathrm{nM})$.

Table 1 Recoveries of the developed SERS method based on 4-MPY functionalized AgNPs for the detection of $\mathrm{Hg}^{2+}$ in tap water samples $(n=3)$

\begin{tabular}{lclc}
\hline Sample & Added $(\mathrm{nM})$ & Found $(\mathrm{nM})$ mean \pm SD & Recovery (\%) \\
\hline 1 & 2 & $1.89 \pm 0.73$ & 94.50 \\
2 & 4 & $4.34 \pm 0.34$ & 108.50 \\
3 & 8 & $7.96 \pm 0.21$ & 99.46 \\
4 & 10 & $9.52 \pm 1.23$ & 95.34 \\
\hline
\end{tabular}

four spiked concentration levels. The experimental results clearly confirmed that this method was potentially applicable for the detection of $\mathrm{Hg}^{2+}$ in real samples.

\section{Conclusions}

In summary, a simple, rapid, and sensitive SERS nanosensor for detection of $\mathrm{Hg}^{2+}$ was developed based on 4-MPY functionalized AgNPs in the presence of spermine. By taking advantage of the redox-regulated surface chemistry between $\mathrm{Hg}^{2+}$ and AgNPs, satisfactory analytical performance was obtained. The developed method has a wide linear range (1-100 nM) and a high sensitivity $(0.34 \mathrm{nM})$. In addition, this method exhibited a good selectivity for $\mathrm{Hg}^{2+}$ over other interfering metal ions and was successfully applied to analysis of $\mathrm{Hg}^{2+}$ in tap water samples. With the appropriate choice of nanomaterials and Raman reporter molecules, especially the smart utilization of specific interactions between targets and substrates, such a sensing strategy can provide great potential for construction of SERS sensors toward various heavy metals.

\section{Acknowledgements}

The authors gratefully acknowledge financial support of National Natural Science Foundation of China (21275158), the 
100 Talents Program of the Chinese Academy of Sciences, and the Science and Technology Development Plan of Yantai City of China (2011071).

\section{Notes and references}

1 J. R. Miller, J. Rowland, P. J. Lechler, M. Desilets and L. C. Hsu, Water, Air, Soil Pollut., 1996, 86, 373.

2 I. Hoyle and R. D. Handy, Aquat. Toxicol., 2005, 72, 147.

3 P. B. Tchounwou, W. K. Ayensu, N. Ninashvili and D. Sutton, Environ. Toxicol., 2003, 18, 149.

4 F. X. Han, W. Dean Patterson, Y. J. Xia, B. B. Maruthi Sridhar and Y. J. Su, Water, Air, Soil Pollut., 2006, 170, 161.

5 G. Aragay, J. Pons and A. Merkoci, Chem. Rev., 2011, 111, 3433.

$6 \mathrm{~J}$. Gomez-Ariza, F. Lorenzo and T. Garcia-Barrera, Anal. Bioanal. Chem., 2005, 382, 485.

7 Y. L. Yu, Z. Du and J. H. Wang, J. Anal. At. Spectrom., 2007, 22, 650.

8 B. Fong, W. Mei, T. S. Siu, J. Lee, K. Sai and S. Tam, J. Anal. Toxicol., 2007, 31, 281.

9 J. H. Li, W. H. Lu, J. P. Ma and L. X. Chen, Microchim. Acta, 2011, 175, 301.

10 A. R. Timerbaev, Chem. Rev., 2013, 113, 778.

11 L. Chen, T. T. Lou, C. W. Yu, Q. Kang and L. X. Chen, Analyst, 2011, 136, 4770.

12 D. B. Liu, W. W. Chen, J. H. Wei, X. B. Li, Z. Wang and X. Y. Jiang, Anal. Chem., 2012, 84, 4185.

13 N. Zhou, J. H. Li, H. Chen, C. Y. Liao and L. X. Chen, Analyst, 2013, 138, 1091.

14 A. E. Lanterna, E. A. Coronado and A. M. Granados, J. Phys. Chem. C, 2012, 116, 6520.

15 X. Xue, F. Wang and X. Liu, J. Am. Chem. Soc., 2008, 130, 3244.
16 M. Rex, F. E. Hernandez and A. D. Campiglia, Anal. Chem., 2006, 78, 445.

17 T. T. Lou, Z. P. Chen, Y. Q. Wang and L. X. Chen, ACS Appl. Mater. Interfaces, 2011, 3, 1568.

18 A. Campion and P. Kambhampati, Chem. Soc. Rev., 1998, 27, 241.

19 L. Guerrini and D. Graham, Chem. Soc. Rev., 2012, 41, 7085.

20 Y. Q. Wang, B. Yan and L. X. Chen, Chem. Rev., 2013, 113, 1391.

21 J. L. Li, L. X. Chen, T. T. Lou and Y. Q. Wang, ACS Appl. Mater. Interfaces, 2011, 3, 3936.

22 Y. Y. Zhu, H. Kuang, L. G. Xu, W. Ma, C. F. Peng, Y. F. Hua, L. B. Wang and C. L. Xu, J. Mater. Chem., 2012, 22, 2387.

23 X. H. Jiang, Y. C. Lai, M. Yang, H. Yang, W. Jiang and J. H. Zhan, Analyst, 2012, 137, 3995.

24 W. Ma, M. Z. Sun, L. G. Xu, L. B. Wang, H. Kuang and C. L. Xu, Chem. Commun., 2013, 49, 4989.

25 L. X. Chen, X. L. Fu and J. H. Li, Nanoscale, 2013, 5, 5905.

26 G. Q. Wang and L. X. Chen, Chin. Chem. Lett., 2009, 20, 1475.

27 W. Ren, C. Zhu and E. Wang, Nanoscale, 2012, 4, 5902.

28 H. S. Jung, K. Kim and M. S. Kim, J. Mol. Struct., 1997, 407, 139.

29 J. Hu, B. Zhao, W. Xu, B. Li and Y. Fan, Spectrochim. Acta, Part A, 2002, 58, 2827.

30 B. Pergolese, M. Muniz-Miranda and A. Bigotto, J. Phys. Chem. B, 2004, 108, 5698.

31 C. Y. Wu, W. Y. Lo, C. R. Chiu and T. S. Yang, J. Raman Spectrosc., 2006, 37, 799.

32 G. Wang, C. Lim, L. Chen, H. Chon, J. Choo, J. Hong and A. J. deMello, Anal. Bioanal. Chem., 2009, 394, 1827.

33 J. C. Fraire, L. A. Pérez and E. A. Coronado, J. Phys. Chem. C, 2013, 117, 23090.

34 Y. F. Wang, Z. H. Sun, H. L. Hu, S. Y. Jing, B. Zhao, W. Q. Wu, C. Zhao and J. R. Lombardi, J. Raman Spectrosc., 2007, 38, 34. 